\title{
Diagnostic work-up in peripheral neuropathy: an analysis of 171 cases
}

\author{
Doris Lubec, Wolf Müllbacher, Josef Finsterer, Bruno Mamoli
}

\begin{abstract}
Summary
This study was set up to evaluate retrospectively the efficacy of a standard diagnostic procedure, including noninvasive and invasive (spinal tap, nervel muscle biopsy) investigations, in the diagnosis of peripheral neuropathy. The medical records of 171 in-patients with the final diagnosis of peripheral neuropathy of determined or undetermined cause were reviewed and each individual diagnostic work-up was analysed. Basic investigations included the patient's history, a clinical examination and basic laboratory tests. Depending on the individual presentation, course, and severity, further noninvasive and invasive examinations were added according to the department's standard diagnostic procedure. The aetiology could be clarified in 124 patients $(73 \%)$ and remained unclear in 47 cases. Excluding cases with acute and chronic inflammatory polyneuropathy $(n=14)$, the number of idiopathic peripheral neuropathies dropped to 33 . Non-invasive investigations were sufficient to reveal the underlying aetiology in 114 cases ( $83 \%)$. It is concluded that, with the application of a standard procedure for the diagnosis of peripheral neuropathy, the aetiology can be clarified in $81 \%$ of patients. In the other $19 \%$ of patients the aetiology remains idiopathic. In the majority of cases, noninvasive investigations were sufficient for diagnosis.
\end{abstract}

Keywords: neuromuscular disorders; diagnosis; electrophysiology; peripheral neuropathy

Peripheral neuropathies are among the most common neurological diseases. They are either inherited or acquired and are often associated with various systemic disorders. ${ }^{2}$ Since they may lead to major disability and handicap, a careful diagnostic clarification is a prerequisite for appropriate treatment. $^{3-5}$ Recent studies have shown that, by costly and partially invasive investigations, the proportion of unclassified (idiopathic) peripheral neuropathies can be substantially decreased. ${ }^{246-8}$ Detailed diagnostic procedures for evaluation of peripheral neuropathies have been proposed, but a uniform, systematic, diagnostic approach is not available. ${ }^{9-12}$ In order to elaborate guidelines for an efficient diagnostic work-up of peripheral neuropathies, we reviewed the clinical, labora- tory, electrophysiological and nerve/muscle biopsy findings of 171 consecutive in-patients with an established diagnosis of peripheral neuropathy referred to our department during a 5-year period.

\section{Materials and methods}

We evaluated the medical records of all patients who attended the Department of the Neurological Hospital Rosenhügel, Vienna, between 1992 and 1996 and were discharged with a final diagnosis of a peripheral neuropathy. Included were 171 patients (97 males, 74 females), aged 28 to 93 years, who were referred by general practitioners or specialists for suspected peripheral neuropathy or other neurological disorders. In all subjects, a detailed general and neurological history had been taken and all had undergone a full clinical examination and basic laboratory investigations that included erythrocyte sedimentation rate, serum glucose, glycated haemoglobin, C-reactive protein, a complete blood cell count, cholesterol, triglycerides, liver enzymes, bilirubin, thyroid function tests, serum electrophoresis, creatinine, urea nitrogen, VDRL and FTA-ABS, HIV and urine analysis.

Further diagnostic tests (additional laboratory tests, electrophysiological examinations, and invasive investigations (spinal tap, nerve/ muscle biopsy)) were ordered individually, depending on the patient's history, the clinical examination, and the basic laboratory tests. Additional laboratory tests included estimation of vitamin B1, B6, B12, folic acid, a Schilling test, immunoelectrophoresis, antinuclear antibodies, antineutrocyte-cytoplasm antibodies, circulating immune complexes, rheumatoid factors, antibodies against ganglioside GM1 and tumour markers (carcino-embryonic antigen, alpha-fetoprotein, carcinoid antigen 19-9, $125,15-3$, and $72-4$, tissue polypeptide antigen, neuron-specific enolase, prostate-specific antigen, prostatic acid phosphatase, squamous cell carcinoma, $\beta$-human chorionic gonadotropin, calcitonin and mucous-cell antigen).

Electrophysiological investigations included nerve conduction studies and needle electromyography according to established guidelines. ${ }^{13}$ For motor nerve conduction studies, surface electrodes were used. For antidromic and orthodromic sensory nerve conduction studies, surface ring electrodes and unipolar needle electrodes were used, respectively. Evaluated variables were the distal motor latency, motor and sensory nerve conduction 
velocity and amplitude of the compound muscle and nerve action potential. The variables were considered abnormal when they exceeded the mean $\pm 2 S D$, established in our laboratory. A demyelinating neuropathy was assumed if the conduction velocity was $<28 \mathrm{~m} / \mathrm{s}$ and the compound muscle action potential $>1 \mathrm{mV}$. An axonal neuropathy was assumed if the compound muscle action potential was below $1 \mathrm{mV}$ and the nerve conduction velocity $>28 \mathrm{~m} / \mathrm{s}^{14}$ Needle electromyography was carried out with concentric needle electrodes qualitatively (assessment of insertion activity, spontaneous activity, interference pattern) and quantitatively (calculation of mean motor unit action potential duration out of 20 potentials).

Cerebrospinal fluid (CSF) examination included tests for total protein, lactate, glucose, protein electrophoresis, total cell count, cell analysis and search for antibodies against borreliae, bacteria and neurotropic viruses. Biopsies were taken from the sural nerve and gastrocnemial muscle, for morphological, histochemical, immunohistochemical and biochemical analyses.

\section{Results}

Purely motor manifestations were found in 23 cases, purely sensory manifestations in 20 , and both sensory and motor involvement in 128 cases. The neuropathy was proximally accentuated in 10 cases (diabetes mellitus, vitamin B deficiency, inflammatory polyarthritis, idiopathic) and distally pronounced in the remaining cases. Distribution of the lesion was asymmetric in five cases (diabetes mellitus, idiopathic) and symmetric in the remaining 166 patients. The time course was acute $(<3$ weeks) in 10 cases, subacute ( $<3$ months) in 41 cases and chronic ( $>3$ months) in the remaining 120 cases. Painful sensations were reported from 58 patients (diabetes, idiopathic, alcohol, gammopathy, paraneoplastic, borreliosis, sarcoidosis, hyperthyroidism, hypothyroidism, Sneddon's syndrome, Crohn's disease, chronic polyarthritis, solvents, benign gammopathy and paraproteinaemia).

Additional laboratory tests were performed in the following frequencies: vitamin B1 $(n=21), \mathrm{B} 6(\mathrm{n}=18), \mathrm{B} 12(n=111)$, folic acid $(n=113)$, Schilling test $(n=108)$, immunoelectrophoresis $(n=114)$, antinuclear antibodies $(n=98)$, antineutrocyte-cytoplasm antibodies $(n=79)$, circulating immune complexes $(n=85)$, rheumatoid factors $(n=69)$, antibodies to ganglioside GM1 $(n=6)$ and tumour markers $(n=114)$. The results of these investigations are given in the table.

Electrophysiological investigations were carried out in 147 patients. Nerve conduction studies were performed in 145 patients and electromyography in 59 patients. Electroneurography was abnormal in 141 patients and electromyography was abnormal in 40 patients. Demyelination was found in 17 cases (acute inflammatory demyelinating polyneuropathy (Guillain-Barre syndrome (GBS)), chronic inflammatory demyelinating polyneuropathy (CIDP), diabetes mellitus, idiopathic), an ax-
Table Frequency of causal factors in 124 clarified peripheral neuropathies and 14 patients with acute and chronic inflammatory demyelinating polyneuropathy (GBS, CIDP)

\begin{tabular}{lll}
\hline Cause & Single $^{\star}$ & Additional $^{\star *}$ \\
\hline Diabetes mellitus & 26 & 14 \\
Alcohol & 20 & 9 \\
Vitamin B1 deficiency & 0 & 17 \\
Vitamin B6 deficiency & 5 & 8 \\
Vitamin B12 deficiency & 7 & 5 \\
GBS & 9 & 0 \\
Gammopathy/paraproteinemia & 6 & 3 \\
Hypothyroidism & 5 & 2 \\
Borreliosis & 6 & 1 \\
Paraneoplasia & 4 & 3 \\
CIDP & 5 & 0 \\
Hereditary & 3 & 2 \\
Hyperthyroidism & 3 & 1 \\
Critical illness & 2 & 1 \\
Vasculitis & 3 & 0 \\
Sarcoidosis & 1 & 0 \\
Vincristine & 1 & 0 \\
Azathioprine & 1 & 0 \\
Refsum's disease & 1 & 0 \\
Sneddon's syndrome & 1 & 0 \\
Ehlers-Danlos syndrome & 1 & 0 \\
Crohn's disease & 1 & 0 \\
Inflammatory polyarthritis & 1 & 0 \\
Folic acid deficiency & 1 & 0 \\
Solvents & 1 & 0 \\
\hline * & &
\end{tabular}

$\star$ : number of patients in whom the given cause was the only one; $\star \star$ : number of patients in whom, in addition to the given cause, at least one other was found

onal lesion in 16 cases (diabetes mellitus, sarcoidosis, hyperthyroidism, idiopathic), and a mixed lesion in the remaining tested cases. In 19 patients with normal electromyography, the electroneurography confirmed the presence of a peripheral neuropathy. Both electroneurographic and electromyographic investigations were performed in 57 patients, ascertaining the diagnosis of peripheral neuropathy in each case.

Spinal taps were performed in 74 patients. CSF investigation revealed neuroborreliosis in seven patients and was compatible with GBS in nine and with CIDP in five cases. A biopsy of the sural nerve and/or of the gastrocnemial muscle was carried out in 27 individuals. Twenty-one patients had a combination of nerve and muscle biopsy, four had only nerve, and two only muscle biopsy, respectively. In three subjects, a vasculitis was diagnosed. The remaining 24 biopsies were unspecifically abnormal.

Overall, the underlying cause could be ascertained in 124 cases ( $73 \%$ ). When patients with GBS and CIDP were included $(n=14)$, this number increased to $138(81 \%)$. Noninvasive investigations were sufficient to specify the underlying aetiology in 114 cases (83\%). In 24 cases $(17 \%)$ invasive procedures (spinal tap, nerve/muscle biopsy) were necessary to reach the final diagnosis. In 89 patients, a single aetiology could be specified, while in 34 subjects, more than one cause was detected. The most frequent aetiologies found in our cohort are listed in the table.

\section{Discussion}

A systematic approach to the diagnosis of peripheral neuropathies is important because of the likelihood of finding an underlying treatable cause. Though detailed diagnostic proce- 
dures for the evaluation of peripheral neuropathies have been proposed, a uniform systematic diagnostic approach is still not available. $^{12101516}$ Recent studies have shown that the aetiology can be ascertained in about $80 \%$ of cases, a figure confirmed by the present study. The remaining idiopathic peripheral neuropathies tend to have a relatively good prognosis. $^{5817}$

At the beginning of the diagnostic work-up, a detailed patient and family history was taken. Special attention was paid to symptoms and signs of general diseases, antecedent infections or immunising procedures, alcohol and drug abuse, medical history and possible exposure to industrial or environmental toxins. The exact time-course and the characteristics of the evolution of the neurological symptoms were analysed. The full neurological and general examination then focused on any aspects that have been gleaned from the history. Special attention was paid to the presence of autonomic symptoms, a pes cavus or other skeletal deformity, enlarged nerves, lymph nodes, enlarged liver or spleen, skin lesions, joint deformities, eye disorders or dry mucous membranes. From the patient history and the results of the clinical examination, the type of neuropathy could be assessed in broad terms (ie, symmetric or assymetric, focal, multifocal or generalised, sensory, motor or mixed, proximal, distal or diffuse, whether autonomic involvement was present and whether the neuropathy was painful or not). ${ }^{11} 1820$ When the results of the basic laboratory investigations were available, the underlying aetiology of the neuropathy could be assessed in some individuals as being toxic, alimentary, metabolic, inherited or inflammatory. ${ }^{21-23}$

The selection of additional laboratory investigations in a particular patient was defined by the individual clinical picture. Nevertheless, a 'check-list' of laboratory tests was almost uniformly performed as the next step in the diagnostic work-up, when the basic tests had not been helpful in clarifying the underlying aetiology. A number of useful assays for neuropathies are available, as outlined above. ${ }^{5}{ }^{102}$ However, many of these tests were useful only in selected patients, and thus cannot be recommended as a general screening procedure. Applying these tests, the most common causes of a chronic peripheral neuropathy, such as diabetes mellitus, alcoholism and vitamin-B deficiency, could be ascertained in our patients.

In all tested subjects, electrophysiological investigations were helpful in confirming the presence of a peripheral neuropathy. ${ }^{5} 710$ 13-15 25-27 In particular, they assisted in determining whether the patient had a generalised, multifocal or focal neuropathy, whether it was symmetric or asymmetric, whether both sensory and/or motor fibres were affected, and whether the underlying pathology was an axonal degeneration or a segmental (CIDP or multifocal motor neuropathy) or diffuse (hereditary neuropathy) demyelination. However, electrodiagnostic studies were not performed when the cause and the extent of the neuropathy was clinically evident.
If an aetiologic classification of the neuropathy was not possible after all these non-invasive investigations, invasive investigations (spinal tap, nerve/muscle biopsy) were carried out in more severe and progressive cases. Unfortunately, a lumbar puncture with examination of the CSF was diagnostically unrewarding in most cases of axonal neuropathy. In contrast, it was substantially helpful in the demyelinating peripheral neuropathies and in neuroborreliosis, and helped to exclude another chronic inflammatory genesis in the remaining cases.

If the aetiology of the neuropathy was still unclear, a nerve and muscle biopsy was performed. Nerve biopsy is often regarded as a valuable method for establishing a cause of peripheral neuropathy under specific circumstances. ${ }^{14} 152428-31$ Several investigators advise cautious and selective use of sural nerve biopsy. Others have a more general approach, proposing that the biopsy should be performed to establish the diagnosis of a peripheral neuropathy, to characterise the neuropathy, and to reach a definite pathological diagnosis. ${ }^{2}$ In general, nerve biopsy is reported to be of little diagnostic value in metabolic disorders and nutritional neuropathies, in which the histological findings are non-specific, ${ }^{4}$ stressing the fact that a biopsy should be considered only when the basic diagnostic effort was inconclusive and when detailed laboratory investigations have been completed. Important exceptions are disorders such as vasculitis, CIDP, amyloidosis, sarcoidosis, leprosy and tumour infiltration. Biopsy was also shown to be useful in the identification of inherited myelinopathies and in some axonopathies. ${ }^{16} 32$ In our patients, the biopsy always confirmed the presence of a peripheral neuropathy when the diagnosis had already been established by clinical examination and electrophysiological studies. Except for the vasculopathic neuropathies in three cases, the biopsy was of little diagnostic value in our series, as only unspecific findings were obtained..$^{33}$ Accordingly, biopsy seems to be of limited help in patients with peripheral neuropathy.

By means of the presented diagnostic approach, the underlying aetiology could be specified in a total of 124 patients $(73 \%)$, leaving the remaining 47 patients with a diagnosis of an idiopathic peripheral neuropathy. However, this group of idiopathic peripheral neuropathies also included patients with GBS and CIDP $(n=14)$, in which the underlying causes can rarely be established. Excluding these cases, 33 patients remained with the definite diagnosis of an idiopathic peripheral neuropathy $(19 \%)$. This figure is in agreement with previously published studies (24, 13 and $14 \%)$, in which patients with GBS and CIDP were also excluded from further aetiologic

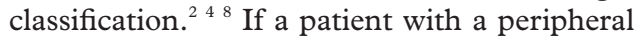
neuropathy is carefully and systematically evaluated, as outlined here, idiopathic peripheral neuropathy will be an uncommon diagnosis, although in some cases such a diagnosis will be inevitable despite a complete diagnostic work-up. Advances in molecular genetics ${ }^{32}$ and 
the immunopathology of peripheral nerve disorders ${ }^{33}$ are likely to reveal the underlying aetiology of some of the peripheral neuropathies that are currently diagnosed as idiopathic in the near future.

In conclusion, we could show diabetes mellitus and alcohol abuse to be the most frequent causes of peripheral neuropathies in our population. The majority $(69 \%)$ of our patients were diagnosed using 'basic' diagnostic tools, and another $20 \%$ could be diagnosed by means of additional laboratory investigations. In the remaining cases $(17 \%)$, invasive procedures (spinal tap, nerve/muscle biopsy) were necessary to reach the final aetiologic classification (GBS, CIDP, neuroborreliosis, vasculitis). Hence, the vast majority of diagnoses could be confirmed using non-invasive procedures. More invasive investigations are of minor importance for clarifying the aetiology of peripheral neuropathies. These findings clearly favour a primarily noninvasive, 'basic' diagnostic approach. Invasive procedures should be performed only in carefully selected individuals. A summary of these diagnostic procedures is given in the box.

We are grateful to the Verein zur Erforschung der Muskelkrankheiten, Vienna, Austria.

\section{Useful diagnostic procedures for clarifying the aetiology of peripheral neuropathies}

\section{STEP 1}

History

Clinical examination

Basic laboratory tests: erythrocyte sedimentation rate, C-reactive protein, fasting blood glucose, glycosilised haemoglobin, creatinine, urea nitrogen, serum electrolytes including calcium and phosphate, liver enzymes including creatine kinase, bilirubin, cholesterol, triglycerides, complete blood cell count, serum protein electrophoresis, thyroid function tests $\left(\mathrm{T}_{3}, \mathrm{~T}_{4}, \mathrm{TSH}\right), \mathrm{VDRL}$ and FTA-ABS, HIV, serum levels of folate and vitamin B12, urine analysis

STEP 2 (in selected patients depending on the results of the basic investigations)

Additional laboratory tests: immunoelectrophoresis and immunofixation, ${ }^{325} 378$ serum levels of vitamin B1, B6, Schilling test, antinuclear antibodies, antineutrocyte-cytoplasm antibodies, circulating immune complexes, rheumatoid factors, antibodies against ganglioside, MAG, $\mathrm{Ro}, \mathrm{Xi}$ or $\mathrm{Hu},{ }^{34-36} 3940$ screening for occult malignancy, cryoglobulins, porphyrins, phytanic acid, long chain fatty acids, heavy metals, molecular genetic search for mutations in the PMP22, PMP0, connexin-32, EGR2, thyrosinkinase A, transthyretin, gelsoline and apolipoprotein A genes

Electroneurography/electromyography

Lumbar puncture

Biopsy
1 Chalk CH. Acquired peripheral neuropathy. Neurol Clin 1997;15:501-24.

2 Dyck PJ, Oviatt RF, Lambert EH. Intensive evaluation of referred unclassified neuropathies yields improved diagnosis. Ann Neurol 1981;10:222-6.

3 Latov N. Pathogenesis and therapy of neuropathies associated with monoclonal gammopathies. Ann Neurol 1995;37(suppl 1):32-42.

4 McLeod JG, Tuck RR, Pollard JD, Cameron JC, Walsh JC. Chronic peripheral neuropathy of undetermined cause. $\mathscr{f}$ Neurol Neurosurg Psychiatry 1984;47:530-5.

5 McLeod JG. Investigation of peripheral neuropathy. $7 \mathrm{Neu}$ rol Neurosurg Psychiatry 1995;58:274-83.

6 Hughes RAC. Chronic peripheral neuropathy of undetermined cause. In: Matthews WB, ed. Handbook of clinical neurology. Neuropathies. Amsterdam: Elsevier, 1987; pp 52941.

7 Notermans NC, Wokke JHJ, Franssen H, et al. Chronic idiopathic peripheral neuropathy presenting in middle or old age: A clinical and electrophysiological study of 75 patients. F Neurol Neurosurg Psychiatry 1993;56:1066-71.

8 Prineas JW. Peripheral neuropathies of undetermined cause. Acta Neurol Scand 1970;44(suppl):1-72.

9 Cohen JA, Gross KF. Peripheral neuropathy: causes and management in the elderly. Geriatrics 1990;45:21-34.

10 Griffin JW, Hsieh S, McArthur JC, Cornblath DR. Laboratory testing in peripheral nerve disease. Neurol Clin 1996;14 119-31.

11 Ludin HP, Tackmann W: Polyneuropathien. Stuttgart, New York: Georg Thieme Verlag, 1984.

12 Thomas PK. Undiagnosed neuropathies: the impact of ancillary investigations. Bailliere Clin Neurol 1996;5:157-70.

13 Kimura J. Nerve conduction studies and electromyography. In: Dyck PJ, Thomas PK, Low PA, Griffin JW, Poduslo JF, eds. Peripheral neuropathy. Philadelphia: WB Saunders, 1993; pp 598-644.

14 Sluga E, Mamoli B, Pateisky K. Vergleichende bioptische und elektrophysiologische Untersuchungen bei Polyneuropathien. Wien Klin Wochenschr 1977;4:113-22.

15 Trush D. Investigations of peripheral neuropathy. Br f Hosp Med 1992;48:13-22.

16 Barohn RJ. Approach to peripheral neuropathy and neuronopathy. Semin Neurol 1998;18:7-18.

17 Koenig F, Neundoerfer B, Koempf D. Polyneuropathien im höheren Lebensalter. Dtsch Med Wochenschr 1984;109:735höh 7 .

18 Said G. Diabetic neuropathy: an update. F Neurol 1996;243: 431-40.
19 Steiner I, Kidron D, Soffer D, Wirguin I, Abramsky O. Sensory peripheral neuropathy of vitamin B 12 deficiency: a primary demyelinating disease? $\mathcal{F}$ Neurol 1988;235:163-4.

20 Wokke JHJ, van Dijk GW. Sensory neuropathies including painful and toxic neuropathies. F Neurol 1997;244:209-21.

21 Davies L, Spies JM, Pollard JD, McLeod JG. Vasculitis confined to peripheral nerves. Brain 1996;119:1441-8.

22 Emeryk-Szajewska B, Badurska B, Kostera-Pruszczyk A. Electrophysiological findings in hereditary motor and sensory neuropathy type I and II-a conduction velocity study. Electromyogr Clin Neurophysiol 1998;38:95-101.

23 Hawke SHB, Davies L, Pamphlett R, Guo Y-P, Pollard JD, McLeod JG. Vasculitic neuropathy. Brain 1991;114:217590.

24 Berek K, Margreiter J, Willeit J, Berek A, Schmutzhard E, Mutz NJ. Peripheral neuropathies in critically ill patients: a prospective evaluation. Intens Care Med 1996;22:849-55.

25 Kelly JJ Jr. The electrodiagnostic findings in peripheral neuropathies associated with IgM monoclonal gammopathis. Muscle Nerve 1990;13:1113-7.

26 Shefner JM. The use of sensory action potentials in the diagnosis of peripheral nerve disease. Arch Neurol 1990;47: $341-7$.

27 Mitsumoto H, Wilbourn AJ. Causes and diagnosis of sensory neuropathies: a review. F Clin Neurophysiol 1994;11: 553-67.

28 Argov Z, Steiner I, Soffer D. The yield of sural nerve biopsy in the evaluation of peripheral neuropathies. Acta Neurol Scand 1989;79:243-5.

29 Chia L, Fernandez A, Lacroix C, Adams D, Plante V, Said $\mathrm{G}$. Contribution of nerve biopsy findings to the diagnosis of disabling neuropathy in the elderly. A retrospective review of 100 consecutive patients. Brain 1996;119:1091-8.

30 Logigian EL, Kelly JJ, Adelmann S. Nerve conduction and biopsy correlation in over 100 consecutive patients with suspected peripheral neuropathy. Muscle Nerve 1994;17:101pected 20 .

31 Vallat JM. Electron microscopy as a tool to diagnose neuropathies. Bailliere Clin Neurol 1996;5:143-56.

32 Reilly MM. Genetically determined neuropathies. 7 Neurol 1998;245:6-13.

33 van der Meche FG, van Doorn PA. Future developments in the treatment of immune-mediated polyneuropathis. Eur Neurol 1997;38:230-37.

34 Finsterer J, Muellbacher W, Halbmayer WM, Fischer M, Mamoli B. Anti-GM1 antibodies in peripheral neuropathies of unknown origin. 7 Clin Pathol 1996;49:422-5. 
35 Gorson KC, Ropper AH, Palmetshofer AK, Weinstein R. Prevalence of polyclonal gammopathy in peripheral neuropathy. Neurology 1997;49:1747.

36 Smith PS, Posner JB. Paraneoplastic peripheral neuropathy. Bailliere Clin Neurol 1995;4:443-68.

37 Nemni R, Gerosa E, Piccolo G, Merlini G. Neuropathies associated with monoclonal gammapathies. Haematologica 1994;79:557-66.
38 Smith IS. The natural history of chronic demyelinatingneuropathy associated with benign IgM paraproteinemia. A clinical and neurophysiological study. Brain 1994;117:949-57.

39 Voltz RD, Graus F, Posner JB, Dalmau J. Paraneoplastic encephalomyelitis: an update of the effects of the anti-Hu immune response on the nervous system and tumour. $f$ Neurol Neurosurg Psychiatry 1997;63:133-6.

40 Smith DR, Weiner HL. Immunologic aspects of neurologic and neuromuscular diseases. $\mathcal{F A M A}$ 1997;278:1956-61.

\section{Medical Anniversary}

\section{Ludwig van Beethoven, 17 December 1770}

Ludwig van Beethoven (1770-1827) was born in Bonn but the family moved to Vienna in 1792. There is still considerable controversy over his mysterious illnesses. The deafness has been attributed to Paget's disease and auditory neuropathic otosclerosis whereas others have extended it into a multisystem systemic disorder due to systemic lupus erythematosus, sarcoidosis or Whipple's disease. Gastroenterologists have argued in favour of ulcerative colitis, Crohn's enteritis, chronic active hepatitis or alcoholic cirrhosis.

The reader is invited to develop his own diagnosis based on these features - deafness, chronic diarrhoea, abdominal pain, arthralgias and weight loss. He died from pneumonia, extensive dropsy, jaundice and terminal coma. Professor Karl von Rokitansky carried out an autopsy on 28 March 1827, but the features were non-specific and hence the continuing conjecture about his ill-health. - DG Fames 